\title{
Pollster Corner
}

\section{NEWS}

\section{Umfragedaten zu den Landtagswahlen 2011 verfügbar}

Nach dem Superwahljahr 2009 wartet auch 2011 mit insgesamt sieben Landtagswahlen auf. Zu den Landtagswahlen in Hamburg, Sachsen-Anhalt, Baden-Württemberg, RheinlandPfalz und Bremen hat die German Longitudinal Election Study (GLES) nun Umfragedaten veröffentlicht. Hierfür wurden in jedem der betreffenden Bundesländer etwa 500 Personen anhand eines Online-Fragebogens befragt. Im Vordergrund stehen neben aktuellen landespolitischen Themen vor allen Dingen Unterschiede in der Bewertung von Landes- sowie Bundesparteien und -politikern. Hierdurch wird beispielsweise eine genaue Untersuchung des Einflusses von landesund bundespolitischen Faktoren auf Landtagswahlentscheidungen ermöglicht. Alle Datensätze sind nach einer Registrierung über www.gesis.org/wahlen/gles/daten/ kostenfrei zu beziehen. Umfragedaten zu den Landtagswahlen in Berlin und Mecklenburg-Vorpommern folgen in Kürze. (EB)

\section{Wahlanalysen von Forschungsgruppe Wahlen und Infratest dimap zu den bisherigen Landtagswahlen 2011 erschienen}

Wie zu jeder Landtagswahl haben Forschungsgruppe Wahlen e.V. und Infratest dimap auch zu den Wahlgängen in Hamburg, Sachsen-Anhalt, Baden-Württemberg, Rheinland-Pfalz und Bremen zusammenfassende Wahlanalysen erstellt. Basis bilden die im Wahlvorfeld bzw. am Wahltag für das ZDF bzw. die ARD durchgeführten Repräsentativbefragungen. Die Schriftenreihe "Berichte der Forschungsgruppe Wahlen e.V." bzw. der WahlREPORT von Infratest dimap können jeweils als pdf- und/oder Printversionen über die jeweilige Institutshomepage geordert werden: http://www.forschungsgruppe.de/FAQ/Bestellung/ bzw.

https://www.infratest-dimap.de/service/publikationen/ (RH)

\section{Initiative Markt- und Sozialforschung ist eingetragener Verein}

Die im vergangenen Jahr ins Leben gerufene Initiative Marktund Sozialforschung (IMSF) ist seit Juni 2011 ein eingetragener Verein. Seine Aufgabe sieht der Verein darin, Wissenschaft und Forschung in der Markt- und Sozialforschung zu fördern sowie die Öffentlichkeit über Markt- und Sozialforschung zu informieren. Mitglieder sind die vier großen Branchenverbände ADM (Arbeitskreis Deutscher Markt- und Sozialforschung e.V.), BVM (Berufsverband Deutscher Markt- und Sozialforscher e.V.), DGOF (Deutsche Gesellschaft für Onlineforschung e.V.) und ASI (Arbeitsgemeinschaft Sozialwissenschaftlicher Institute e.V.).

Bereits im Mai hatte die Initiative den bundesweit ersten Tag der Marktforschung organisiert, der wegen der großen Resonanz auch im nächsten Jahr stattfinden soll. Nach einer Hörfunk- und Onlinekampagne der Initiative soll demnächst auch im Rahmen einer Printkampagne über die Arbeit der deutschen Markt- und Sozialforschungsinstitute informiert und für die Teilnahme an Befragungen geworben werden. Einen besonderen Stellenwert legt die Initiative darauf, über Unterschiede zwischen Markt- und Sozialforschung auf der einen und Werbung bzw. Verkauf auf der anderen Seite zu informieren. In diesem Zusammenhang rügte die Initiative jüngst die Vertriebspraktiken des Versicherers Ergo, deren Außendienstvertreter als „Türöffner“ die Durchführung von Meinungsumfragen ankündigen.

http://www.deutsche-marktforscher.de/home.html (RH)

\section{FORSCHUNG}

\section{Neuerungen in den Wahlsystemen von Hamburg und Bremen}

Wahlsysteme haben in demokratischen Systemen eine wichtige Funktion: Sie übersetzen den politischen Willen, der sich in der Stimmabgabe der Bürger ausdrückt, in Machtverhältnisse zwischen politischen Parteien. An Wahlsysteme werden daher hohe Anforderungen gestellt. Ein Kriterium für ein gutes Wahlsystem ist dabei, dass es den Bürgern ermöglicht, ihren politischen Willen durch einen gewissen Gestaltungsspielraum bei der Stimmabgabe angemessen auszudrücken.

In Hamburg und Bremen hat man sich nun dazu entschlossen, bei den Bürgerschaftswahlen mehr Gestaltungsspielraum zuzulassen: Die Zahl an Stimmen, die vergeben werden können, wurde erhöht (in Hamburg auf zehn, in Bremen auf fünf), zudem können diese Stimmen frei auf Kandidaten unterschiedlicher Parteien und im Fall von Hamburg auch auf unterschiedliche Landeslisten verteilt werden. Für die Wahlforschung stellen diese Neuerungen einerseits eine Herausforderung dar. Der Stimmzettel für die Bremer Bürgerschaftswahl umfasste je nach Wahlkreis etwa 20 Seiten.

Die genaue Entscheidungssituation kann in einer Umfrage folglich nicht mehr nachgestellt werden, wenn man die Befragten nicht abschrecken und zum Abbruch des Interviews verleiten will. Andererseits ergeben sich auch auf Basis relativ einfacher Abfragen der Wahlentscheidung interessante neue Einblicke in das Wählerverhalten, wie sich am Beispiel von Bremen zeigen lässt. Bei einer Online-Befragung der German Longitudinal Election Study zu den Bürgerschaftswahlen in Bremen wurden die Befragten gebeten, ihre fünf Stimmen auf insgesamt acht Parteien zu verteilen (SPD, CDU, Grüne, Linke, FDP, BIW, NPD und „andere Parteien“). Bei dieser Art der Abfrage fehlen folglich die Informationen dazu, welche Kandidaten genau gewählt wurden, dennoch bietet sie Einiges an Analysemöglichkeiten. Wie das geht, zeigt das folgende Beispiel: Auf Basis der Frage, wie die fünf Stimmen auf die Parteien verteilt werden, lässt sich zunächst einmal herausfinden, auf wie viele Parteien von einem Bürger gewählt werden. Diese Information kann man dann beispielsweise mit der Stärke der Parteineigung in Beziehung setzen. Dabei würden 
wir erwarten, dass Befragte, die sich stark mit einer Partei identifizieren, dieser viele oder sogar alle Stimmen geben, während Befragte ohne oder mit einer nur schwachen Parteineigung ihre Stimmen auf mehr Parteien verteilen. Bevor auf die Ergebnisse dieser kleinen Untersuchung eingegangen wird, muss noch einmal deutlich gemacht werden, dass es sich bei der Online-Umfrage nicht um eine repräsentative Befragung handelt. Der Anteil an Wählern, die ihre Stimmen auf eine Partei bündeln oder auf mehrere Parteien verteilen, weicht daher von den offiziellen Zahlen des Landeswahlleiters ab. Zu den Ergebnissen lässt sich nun sagen, dass unsere Vermutung nicht bestätigt wird. In der Abbildung ist die Zahl an gewähl- ten Parteien innerhalb dreier Wählergruppen dargestellt: Befragte mit starker (Gruppe 1), mäßig bis schwacher (Gruppe 2) und ohne Parteineigung (Gruppe 3). Es ist deutlich zu sehen, dass insbesondere zwischen den Befragten mit starker und ohne Parteineigung keine großen Unterschiede in Hinblick auf die Zahl der gewählten Parteien bestehen. Folglich machen einerseits Wähler mit starker Parteibindung von den neuen Möglichkeiten, die das Wahlsystem ihnen bietet, bedingt Gebrauch. Andererseits konzentrieren sich Wähler ohne starke Parteibindungen auf eine oder zwei Parteien, eine gröBere Zahl an Stimmen führt daher nicht notwendigerweise zu einer deutlich höheren Zahl an gewählten Parteien. (EB)

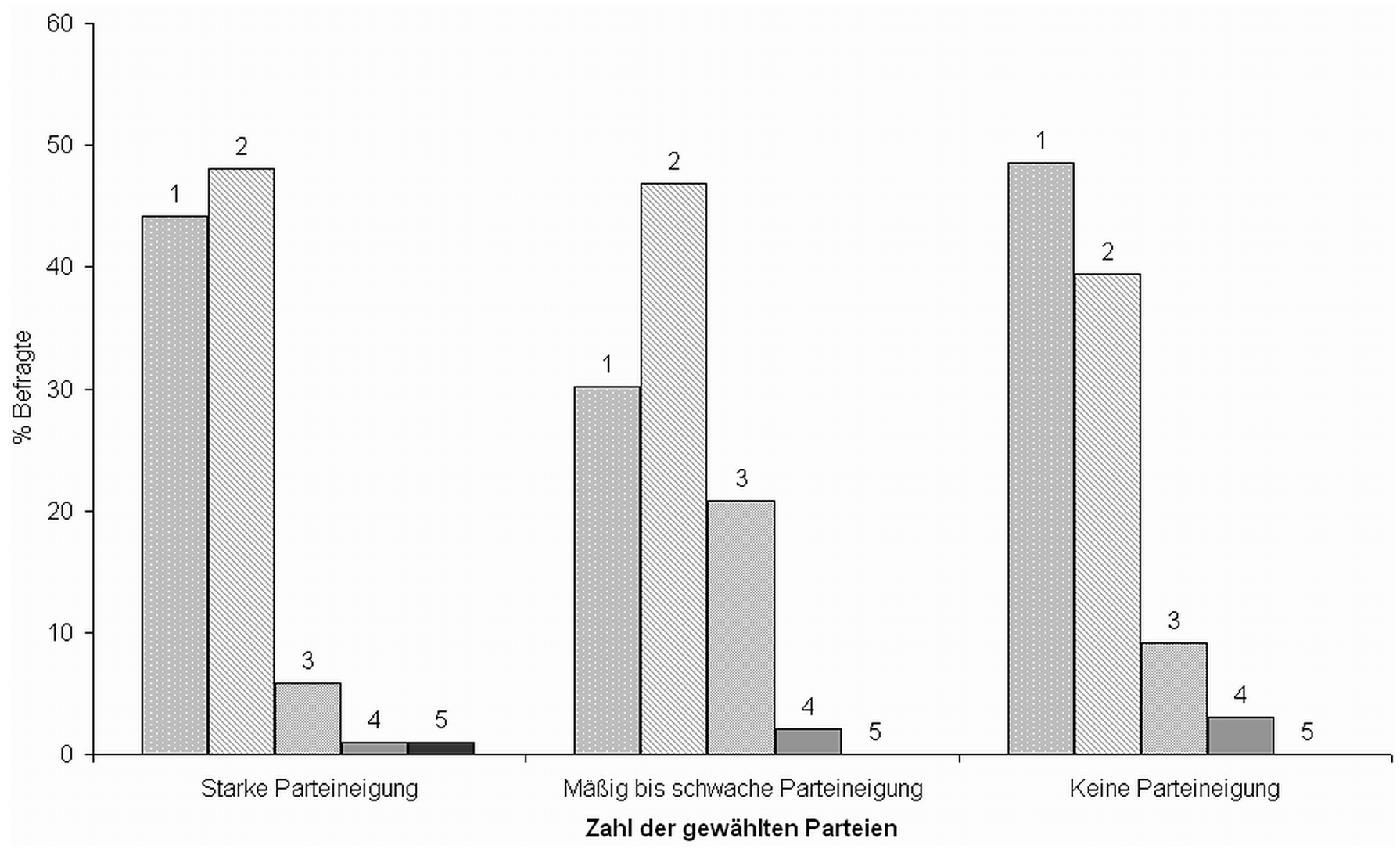

\section{Veranstaltungen}

\section{Messe für Marktforschung Research \& Results 2011: 26. und 27. Oktober in München}

Research \& Results, die deutsche Fachmesse für Markt- und Meinungsforschung, öffnet auch dieses Jahr in München ihre Tore. Bereits zum sechsten Mal präsentieren Markt- und Meinungsforschungsinstitute, Feldorganisationen, Teststudios, Soft- und Hardwareanbieter, Datenerfassungs- und Auswer- tungsservices, Marktforschungsberater, Trendforschungsinstitute und Online-Panel-Provider ihr Leistungsspektrum und stellen in 91 Workshops praxisnahe Forschungslösungen vor. Nähere Informationen unter:

http://www.research-results.de/messe/uebersicht/messeuebersicht.html (RH)

Evelyn Bytzek, Johann Wolfgang-Goethe Universität, Frankfurt, bytzek@soz.uni-frankfurt.de

Roberto Heinrich, Infratest dimap, roberto.heinrich@infratest-dimap.de 